\title{
Estudo de prospecção das patentes de localização sonora componentes da avaliação do processamento auditivo central
}

\author{
Prospective study of sound localization patents componentes of the assessment the central auditory \\ processing
}

Estudio de la prospeccción de patentes de umbicación sonora componentes de la evaluación del procesamiento auditivo central

\section{Resumo}

Objetivo: Foi realizada uma prospecção tecnológica com o objetivo de obter informações sobre a existência de equipamentos que avaliem a localização sonora durante o teste de Processamento Auditivo Central. Metodologia: A busca foi realizada nas bases INPI, Espacenet, Patentscope e Patentinspiration. Foram utilizados os termos "localização", "som", "sonora", "auditiva", "percepção sonora", interligados por operadores boleanos "AND" e "OR”, para formar diversos algoritmos. A pesquisa foi realizada nos campos de título e resumo, utilizando termos em português nas patentes nacionais e em inglês nas patentes internacionais. Resultado e Discussão: Diante das patentes recuperadas, foram consideradas três que se aproximaram do tema pesquisado. Contudo, apenas uma delas serviu para nortear os parâmetros da patente em estudo. Conclusão: Nenhuma patente encontrada se refere à utilização do equipamento com objetivo de avaliar a habilidade de localização durante a avaliação do Processamento Auditivo Central. Isso indica o ineditismo do tema proposto.

Palavras-chave: Localização; Som; Percepção sonora.

\begin{abstract}
Objective: A technological survey was carried out, with the objective of obtaining information about the existence of equipament, with assesses the sound location during the Central Auditory Processing Test. Methodology: The search was carried out using the INPI, Espacenet, Patentscope e Patentinspiration databases. The terms "localization", "sound", "auditive" and "loudness perception" were used, interconnected by boolean operators "AND" e "OR", to form various algorithms. The research was carried out in the title and summart Fields, using terms in Portuguese in national patentes and English in international patents. In veiw of the recorvered patentes. Result and Discussion: In view of the recorvered, 3 were considered to be close to the research topic. However, only of them served to guide the parameters of the patente under study. Conclusion: However, none refers to the use of equipament in order to assess
\end{abstract}


the ability of localization during the assessment of the Central Auditory Processing. This indicates the novelty of the proposed theme.

Keywords: Localization; Sound; Loudness perception.

\section{Resumen}

Objetivo: Se realizo um relevamiento tecnológico com el objetivo de obtener información sobre la existência de equipos, que valora la umbicación del sonido durante la prueba de Procesamiento Auditivo Central. Metodologia: La búsqueda se realizo sobre la base INPI, Espacenet, Patentscope y Patentinspiration. Se utilizaron los términos "ubicacion", "sonido", "auditivo" e "percepción del sonido", interconectado por los operadores booleanos "AND" e "OR", para formar vários algoritmos. La investigación se llevô a cabon em los campos de título y resumen, utilizando términos em português en patetes nacionales y en inglês en patentes internacionales. Resultado e Discusión: A la vista de las patentes recuperadas, 3 se consideraron cercanas al tema investigado. Sin embargo, solo uno de ellos sirvió para orientar los parâmetros de la patente em estúdio. Conclusão: Sin embargo, ninguno se refiere al uso del equipo para evaluar la capacidade de localizacion durante la evaluación del Processamiento Auditivo Central. Esto indica novidade del tema propuesto.

Palabras clave: Ubicación; Sonar; Percepción de sonido.

\section{Introdução}

A identificação da fonte sonora envolve a capacidade de detectar a direção e lugar de origem do som no espaço, sendo definida como localização auditiva (Furst, et al., 2004; Oliveira, et al., 2008a; Geffner, 2019). É uma habilidade da audição que está presente em todos os animais e se desenvolve no ser humano a partir do primeiro semestre de vida, funcionando como um mecanismo de proteção importante para a sobrevivência (Menezes, et al., 2005; Grothe \& Pecka, 2014; Luiz, et al., 2016).

As experiências sonoras vão refinando a competência do indivíduo em perceber a direção espacial do som, informando sobre possíveis riscos de perigo no local em que ele está posicionado (Lima-Gregório, et al., 2010). Ao discriminar as pistas acústicas de frequência, intensidade e duração, o ouvinte pode selecionar o som do meio ambiente, o que é viabilizado pela localização auditiva (Dias \& Pereira, 2008), proporcionando a sensação de segurança do lugar aos videntes e, principalmente, para pessoas acometidas de cegueira (Gerente, et al., 2008). Outra vantagem dessa habilidade de localizar é colaborar na prevenção de acidentes de trabalho, pois as informações sonoras nos planos vertical e horizontal são essenciais para funcionários que utilizam protetores auditivos durante a exposição ao ruído ocupacional (Mondelli, et al., 2010; Menezes, et al., 2014).

Assim, a preservação da audição binaural é uma condição necessária para o bom desempenho da localização acústica, visto que o sujeito utiliza mecanismos monoaurais e binaurais ao mesmo tempo, analisando simultaneamente os estímulos recebidos pelas duas orelhas (Carlile, et al., 2005; Nix, et al., 2006; Lewald, 2002).

Além da necessidade da preservação dos limiares auditivos bilateralmente, a identificação da fonte sonora requer o funcionamento de outras áreas da via auditiva (Ramos \& Pereira, 2005). O tronco cerebral é a primeira estrutura a participar desse processo de localização, que ocorre, mais precisamente, no complexo olivar superior, onde inicia a análise sonora advinda das duas orelhas (Fonseca \& Iorio, 2006). Em seguida, o som se dirige para o lemnisco lateral, o qual mantém a representação binaural do estímulo e, posteriormente, o colículo inferior recebe a informação proveniente das estruturas anteriores, completando o processo dessa habilidade auditiva (Musiek \& Baran, 2020). Estudos também chamam atenção para a participação do córtex auditivo primário, sugerindo que essa estrutura colabora na codificação dinâmica da localização (Risoud, 2018).

Essa determinação da posição da fonte sonora pelas estruturas da via auditiva é possível devido à diferença interaural do estímulo sonoro entre as duas orelhas, ou seja, quando a fonte sonora está de um lado da região cefálica, vai atingir primeiro a orelha mais próxima e leva um breve intervalo de tempo para alcançar a outra orelha (Lima-Gregório, 2010). Associada a esse fator, a diferença de intensidade promove a percepção de um estímulo auditivo elevado na orelha de menor distância da fonte sonora, fazendo com que o estímulo sonoro chegue com uma menor intensidade na orelha contralateral (Menezes, et al., 
2003). Com isso, a diferença no tempo e a diferença de intensidade de recepção do som vão fazer com que a primeira orelha estimulada indique a direção de origem do som (Nishihata, et al., 2012). Pesquisas ainda descrevem que $20 \mathrm{~dB}$ de diferença interaural favorece a localização sonora devido à ocorrência do efeito sombra (Oliveira, et al., 2008a).

Considerado um atenuador do som, o efeito sombra ocorre quando um sinal acústico se move de um lado para o outro na região cefálica, gerando uma diminuição da intensidade sonora, principalmente em altas frequências, devido às barreiras causadas pela cabeça, pavilhão auricular e o tronco do corpo (Tharpe, 2007; Oliveira, et al., 2008b). Essas barreiras são denominadas variações espectrais de transferência anatômica (VETA), que funcionam como atenuador sonoro, pois filtram as informações acústicas, modificando o som original (MENEZES et al., 2004). O ombro também é um exemplo de VETA que contribui com o efeito sombra, pois à medida que apresenta um maior comprimento, promove uma melhor percepção horizontal dos sons com frequência de $2 \mathrm{kHz}$ a $4 \mathrm{kHz}$ (Oliveira, et al., 2008b).

Notavelmente, a localização de diferentes fontes sonoras melhora a discriminação da fala em ambiente com ruído (Mondelli, et al., 2010), o que é importante para o indivíduo compreender a comunicação durante atividades diárias (Oliveira, et al., 2020). Ainda é conhecida a contribuição da habilidade de localizar no processo de atenção seletiva, quando o sistema auditivo é capaz de identificar duas fontes sonoras, reconhecer suas características e, com isso, selecionar a mensagem principal descartando o sinal sonoro desnecessário para o ouvinte (Dias \& Pereira, 2008).

Diante da participação da localização sonora na promoção de outras habilidades auditivas, ela é considerada um prérequisito para o desenvolvimento do PAC (Ahmmed, et al., 2014; Souza, et al., 2015).

As habilidades auditivas são necessárias para que o SNC compreenda a informação ouvida, com eficiência e eficácia (ASHA, 2005; Geffner, 2019). O resultado dessa capacidade do cérebro em compreender a informação auditiva de sons verbais e não verbais é o processamento auditivo central (BSA, 2011). Em outras palavras, o PAC é composto por um conjunto de habilidades neurobiológicas envolvendo mecanismos de comportamentos auditivos capazes de detectar, localizar, discriminar, selecionar, reconhecer padrões auditivos e analisar aspectos de frequência, intensidade e duração do sinal acústico, mesmo quando ele está distorcido ou degradado (ASHA, 2005; AAA, 2010). Esse fenômeno do PAC promove ao SNC a condição em integrar os sons recebidos pelas duas orelhas, selecionar a mensagem principal diante de outros sons interferentes e fechar a ideia da informação auditiva, mesmo em ambientes acusticamente desfavoráveis (CRFa, 2020).

Durante o exame de PAC, a localização auditiva é avaliada por meio de um teste, utilizando um instrumento sonoro (guizo), que é percutido em cinco direções da região cefálica. (Pereira \& Schochat, 2011). Essa avaliação analisa o desempenho das duas orelhas, que recebem informações de formas diferentes, mas ao mesmo tempo realizam juntas a tarefa de interação binaural. Entretanto, há uma escassez de material disponível para utilizar na avaliação dessa habilidade auditiva na prática clínica (Gicov, et al., 2015).

Nesse contexto, fica claro que avaliar a localização auditiva é um fator determinante para a análise do PAC. Entretanto, a ausência de um equipamento que proporcione essa avaliação detalhada tem diminuído a cada dia o uso desse teste de localização auditiva durante o exame de PAC.

Diante da iniciativa de desenvolver uma patente para avaliar a localização auditiva e compor o exame de PAC, surgiu a necessidade de realizar esse estudo, com o objetivo de descrever a busca de anterioridades para mapear patentes e artigos, que detenham tecnologias para produção de equipamentos do teste de localização auditiva do som, que sejam usados na avaliação do processamento auditivo central.

\section{Metodologia}

De acordo com Pereira et al (2018), essa pesquisa é de natureza quantitativa, que requer o levantamento numérico de opiniões sobre o tema em questão. Conforme o suporte metodológico de Gil (2002), o objetivo desse estudo pode ser 
classificado como uma pesquisa exploratória, envolvendo o levantamento bibliográfico de outras experiências nessa área, ou seja, envolve a pesquisas de outras patentes realizadas referentes a temática de localização auditiva.

Dessa forma, trabalho foi fundamentado pela pesquisa nas bases de patentes (Instituto Nacional da Propriedade Industrial-INPI, Espacenent -LATIPAT, Patenscope -WIPO e Patentinspiration), as quais foram utilizadas como ferramenta de prospecção para monitoramento e recuperação dos dados referentes aos pedidos de patentes do teste de localização auditiva utilizados durante a avaliação do PAC.

O período da busca ocorreu de janeiro a abril de 2021. Não houve restrição de data de depósito da patente ou do idioma utilizado nas publicações.

$\mathrm{Na}$ escolha das palavras-chave, foi realizada uma busca inicial do termo macro "localization AND (sound OR auditive) AND central". Entretanto, essa estratégia restringiu as buscas. Diante disso, foram realizadas outras combinações entre os termos, para encontrar os algoritmos adequados capazes de nortear um número maior de patentes referentes a equipamentos de avaliação da localização auditiva. As palavras-chave utilizadas estão descritas na Tabela 1 e estão expostas conforme sua utilização nos respectivos bancos de dados. Assim, foram estabelecidas as mesmas palavras para todas as bases, modificando-as para o inglês durante a pesquisa nos bancos de patentes internacionais.

Tabela 1 - Palavras-chave utilizadas na busca de patentes.

\begin{tabular}{cccc}
\hline INPI & LATIPAT-Espacenet & Patentscope-WIPO & Patentinspiration \\
\hline Localização & Localização & Localization & Localization \\
\hline Sonora & Sonora & Sound & Sound \\
\hline Som & Som & Sound & Sound \\
\hline Auditiva & Auditiva & Auditive & Auditive \\
\hline Percepção sonora & Percepção sonora & Loudness Perception & Loudness Perception \\
\hline
\end{tabular}

Fonte: Autores (2021).

As patentes depositadas no Brasil foram pesquisadas na base de dados do INPI (Instituto Nacional de Propriedade Industrial), como também foi feita a busca no escritório de patentes da América Latina e Espanha, LATIPAT - Espacenet. Durante a pesquisa, utilizou-se as palavras-chave demonstradas na Tabela 1. Para especificar a busca, foi feito um cruzamento a partir da combinação desses termos interligados pelos operadores booleanos AND e OR, resultando nos algoritmos demonstrados na Tabela 3.

Já nas bases de dados internacionais da Patentinspiration e Patetscope, a busca foi norteada pela mesma estratégia estabelecida para as bases nacionais, no entanto foram utilizados os termos em inglês, que estão apresentados na Tabela 1. As combinações dos termos, formando os algoritmos estão disponíveis na Tabela 4, a partir dos quais foram identificadas as produções. Em todas as bases, realizou-se a busca avançada, utilizando os campos "título" e "resumo".

Para direcionar melhor a pesquisa, foi feito um refinamento por meio da Classificação Internacional de Patentes (IPC), a fim de identificar os documentos de patente segundo a área tecnológica, utilizando os códigos de determinação, contidos na Tabela 2, com suas referidas descrições. 
Tabela 2 - Classificação Internacional de Patentes (International Patent Classification - IPC).

\begin{tabular}{cc}
\hline CÓDIGO (IPC) & DESCRIÇÃO DOS CÓDIGOS \\
\hline A61B5/00 & Detecção, medicação ou registro para fins de diagnóstico \\
\hline A61B512 & Audiometria \\
\hline
\end{tabular}

Fonte: Autores (2021).

No mesmo período dessa seleção nas bases de patentes nacionais e internacionais, houve a necessidade de fazer um levantamento de artigos científicos na plataforma da CAPES, a fim de comparar com os resultados das patentes. Nessa linha, a pesquisa foi norteada por diversos termos de forma abrangente, que estão demonstrados na Tabela 6. Com isso, foi possível caracterizar o estado atual da arte.

\section{Resultados e Discussão}

A Tabela 3 apresenta o quantitativo de patentes depositadas no Brasil, América Latina e Espanha, contendo os resultados obtidos por meio da busca nas bases de dados INPI e LATIPAT.

Tabela 3 - Resultados obtidos no INPI e LATIPAT-Espacenet.

\begin{tabular}{lll}
\hline ALGORITMO & INPI & Espacenet \\
LATIPAT \\
\hline localização AND (sonora OR auditiva) AND central & 0 & 0 \\
\hline localização AND som & 2 & 1 \\
\hline localização AND sonora & 2 & 4 \\
\hline localização AND auditiva & 1 & 1 \\
\hline localização AND (sonora OR auditiva) & 38 & 5 \\
\hline localização AND sonora OR auditiva & 38 & 157 \\
\hline localização AND auditiva OR percepção auditiva & 2 & 2 \\
\hline localização OR percepção sonora & 961 & 9 \\
\hline localização auditiva OR percepção sonora & 6 & 5 \\
\hline
\end{tabular}

Fonte: Autores (2021).

Após a leitura dessas patentes na íntegra e exclusão daquelas em duplicidade, foi obtido o quantitativo de patentes referente a localização auditiva, os quais estão expostos na Tabela 3. Dentre as patentes referentes a localização auditiva, três pedidos de proteção patentária, contidas nessas duas bases estão relacionadas com a proposta do nosso estudo (Andrade, et al., 2017; Macambira, et al., 2018; Mauricy, et al., 2002).

A patente de Andrade, et al. (2017), apesar de apresentar um tema que se aproxima ao assunto do nosso estudo, tem o objetivo divergente. Os autores propõem a utilizarum kit de fones em salas de aula, para ensino do mecanismo de localização, minimizando os efeitos do ruído desse ambiente e favorecendo a compreensão da fala.

Dentre as patentes também foi identificado o produto de Macambira, et al. (2018), que se trata de um equipamento para avaliar a quantidade de audição da criança por meio da mudança de comportamento mediante a apresentação do estímulo sonoro. Dessa forma, entende-se que o objetivo desse produto está mais direcionado para detectar perda auditiva na população infantil. 
A patente de Mauricy, et al. (2002) demonstrou objetivo de avaliar a localização sonora nos diversos planos espaciais. Visto que a finalidade de avaliar a localização foi similar ao nosso estudo, essa patente serviu para nortear a construção da nossa propriedade intelectual. Contudo, o produto identificado tem o objetivo de verificar o efeito do ambiente reverberante na localização auditiva e não é utilizado na bateria de exames do PAC, o que diverge da nossa patente em estudo.

A Tabela 4 mostra os resultados da pesquisa feita nas bases Patentscope e Patentinspiration, para os algoritmos criados.

Tabela 4 - Resultados obtidos na Patentscope-WIPO e Patentinspiration.

\begin{tabular}{lcc}
\hline PALAVRAS-CHAVE & $\begin{array}{c}\text { PATENTSCOPE } \\
\text { WIPO }\end{array}$ & PATENTINSPIRATION \\
\hline localization AND auditive & 73 & 838 \\
\hline localization AND auditive AND central & 37 & 87 \\
\hline $\begin{array}{l}\text { localization AND (sound OR auditive) } \\
\begin{array}{l}\text { localization AND sound OR loudness AND } \\
\text { perception }\end{array}\end{array}$ & 123 & 27 \\
\hline $\begin{array}{l}\text { localization AND auditory OR loudness AND } \\
\text { perception }\end{array}$ & 1953 & 74 \\
\hline
\end{tabular}

Fonte: Autores (2021).

O resultado na Tabela 4 demonstrou que o número de patentes depositadas no mercado internacional foi maior que a quantidade de criações nacionais. Entretanto, durante a leitura das patentes citadas na Tabela 4, foi detectado que as inovações em localização não se restringiram apenas a questões auditivas. É importante ressaltar que as patentes encontradas incluíram produtos com outros objetivos de localização, ou seja, o termo de localização foi usado no sentido de identificar a posição de objetos ou pessoas em um determinado lugar, como também de identificação/localização de endereços mais utilizadas nos casos de GPS. E ainda, em outros produtos, o termo "localização" foi usado no sentido de identificar personagens e/ou sons em jogos eletrônicos. Ademais, foram identificadas algumas patentes que utilizaram produtos para localização auditiva do som, corroborando com o estudo em questão, mas com objetivo divergente por não ter a intenção de utilizar o produto em testes de PAC e nem de captação de respostas eletrofisiológicas.

Na base da Patentinspiration também foi possível delinear o atual cenário mundial e tecnológico acerca do assunto de localização auditiva. A Figura 1 mostra a distribuição dos resultados dos países que depositaram o maior número de patentes no assunto de localização auditiva. 
Research, Society and Development, v. 10, n. 8, e27910817333, 2021

(CC BY 4.0) | ISSN 2525-3409 | DOI: http://dx.doi.org/10.33448/rsd-v10i8.17333

Figura 1 - Resultado de patentes por países obtido com algoritmo "localization AND auditive".

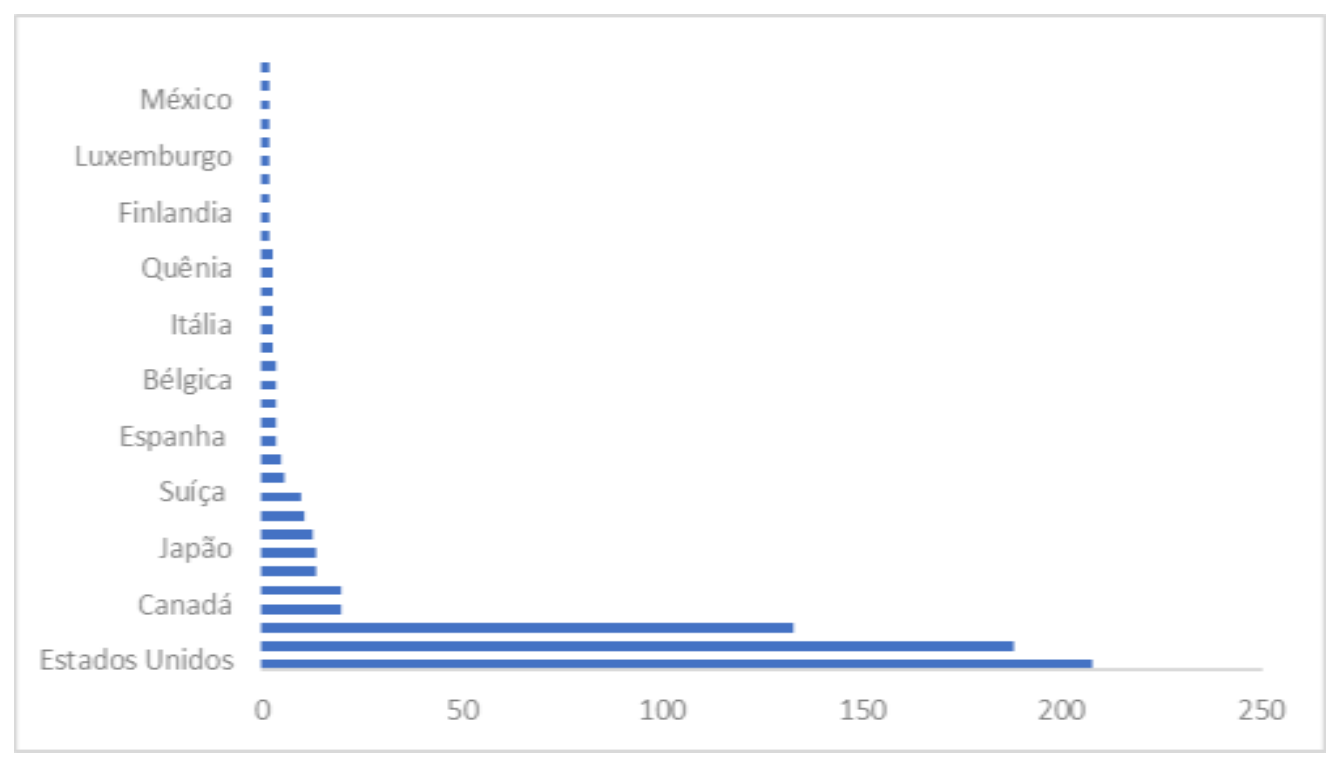

Fonte: Autores (2021).

No ranking mundial, os Estados Unidos da América se destacaram como o maior depositante de patentes em localização. Os dados ainda evidenciam um claro domínio da tecnologia em três países: EUA, China e França; com perspectiva de maior produção nesse setor. Esse domínio se reflete não apenas no número de patentes, mas também para inventores e depositantes. A Figura 2 apresenta o cenário global de patentes depositadas por ano de todos os países.

Figura 2 - Evolução anual do número de patentes no cenário mundial.

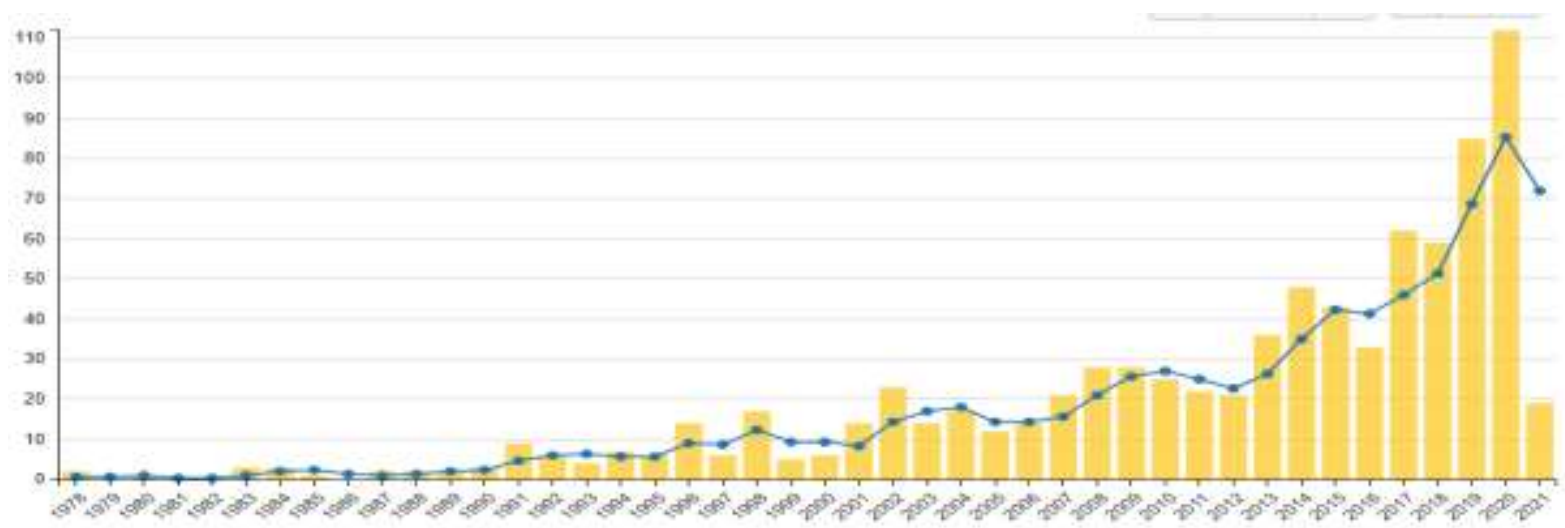

Fonte: Patentinspiration (2021)

Ao fazer o levantamento de datas de depósito dessas patentes por ano, verificou-se que em 1991 inicia-se um momento de crescimento ascendente de publicações. O ano de maior depósito de patentes em localização foi 2020 , com o total de 110. Esse período marca um importante momento de desenvolvimento tecnológico para produção destes produtos.

A Tabela 5 demonstra os códigos da Classificação Internacional de Patentes (IPC - Internacional Patente Classification) correspondente aos respectivos artigos relacionados com o nosso estudo. 
Tabela 5 - Resultados obtidos na Classificação Internacional de Patentes (IPC).

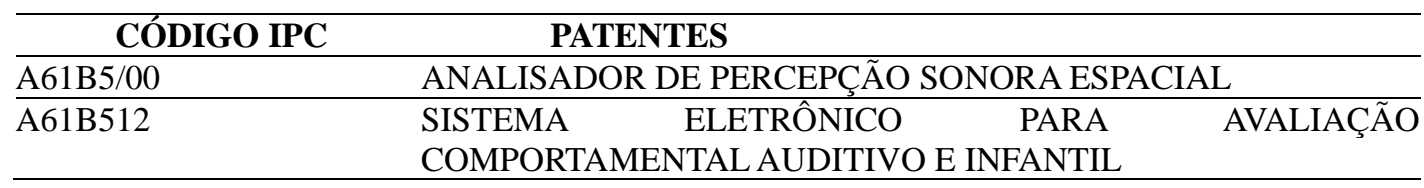

Fonte: Autores (2021).

O resultado do refinamento quanto à Classificação Internacional de Patentes (IPC), demonstrou duas patentes relacionadas com os códigos de base, as quais possuem temas aproximados ao assunto estudado. Assim, um código é referente ao diagnóstico auditivo e o outro utilizado para produtos capazes de avaliar da audição. Por meio dessa busca, foi detectado que os códigos foram utilizados em duas patentes identificadas no INPI e Espacente. No entanto, verificou-se que não há um código específico para o teste de localização auditiva.

O resultado das combinações de palavras-chave e a respectiva quantidade de artigos obtidos nos periódicos CAPES estão demonstrados na Tabela 6.

Tabela 6 - Resultados do número de artigos obtidos com as palavras-chave.

\begin{tabular}{lc}
\hline Palavras-chave & $\begin{array}{c}\text { Periódicos } \\
\text { CAPES }\end{array}$ \\
\hline localização AND som & 544 \\
\hline localização AND sonora & 251 \\
\hline localização AND auditiva & 451 \\
\hline localização AND (sonora OR auditiva) & 283 \\
\hline localização AND audição & 254 \\
\hline localização AND (sonora OR auditiva) AND central & 53 \\
\hline localização AND sonora OR auditiva AND central AND software & 93 \\
\hline \begin{tabular}{l} 
localização AND (sonora OR auditiva) AND central AND (software OR \\
\hline
\end{tabular} & 75 \\
\hline localização AND sonora OR auditiva AND (processamento auditivo central) & \\
\hline
\end{tabular}

Fonte: Autores (2021).

O resultado para as buscas de artigos apresentou um número expressivo de estudos com o tema de testes em localização auditiva utilizados na bateria do PAC. Porém, a grande maioria não teve o objetivo de aplicabilidade de um novo equipamento. Os instrumentos aplicados são estímulos existentes no mercado, os quais são inseridos em computador e emitidos aos pacientes por meio do audiômetro.

A leitura dos artigos na íntegra permitiu verificar que o teste mais utilizado na maioria dos estudos foi o de localização em cinco direções, que é feito com guizo nas cinco direções da região cefálica. É importante destacar o teste de MLD (Masking Level Difference) utilizado na avaliação da interação, o mesmo mecanismo considerado na habilidade de localização auditiva. Não é composto por equipamento, trata-se de estímulo sonoro. O MLD (Masking Level Difference) utiliza sons não verbais na presença do ruído para verificar a vantagem de detecção da fase binaural alterada em relação à fase de condição não alterada. 
Essa prospecção sinaliza a importância do desenvolvimento de uma ferramenta tecnológica para avaliar a localização auditiva. Esse produto, inserido na bateria de testes do processamento auditivo central, vai colaborar com o diagnóstico diferencial da habilidade de localização auditiva, como também vai promover o desenvolvimento de novas pesquisas, que podem permitir tomadas de decisão com melhor direcionamento para tratamento do transtorno do processamento auditivo central. Esse fato também vai despertar o interesse dos profissionais em usar essa tecnologia.

\section{Conclusão}

Neste estudo de prospecção, ficou explícito que nenhuma das patentes existentes teve o objetivo de compor o exame de processamento auditivo central. A escassez de produtos com essa temática reiterou a ideia de que o Brasil tem pouco destaque em publicação de patentes nesse assunto, isso demonstra o ineditismo do tema proposto.

Diante dessa realidade, a perspectiva futura será construção do equipamento de localização auditiva, com a proposta de desenvolver novas pesquisas com esse novo instrumento e colaborar com o diagnóstico diferencial do processamento auditivo central.

\section{Referências}

Ahmemed, A. U., Ahmmed, A. A., Bath, J.R., Ferguson, M. A., Plack, C. J. \& Moore, D. R. (2014). Assessment of children with suspected auditory processing disorder: a fator analysis study. Ear Hear. 35(3), 295-305.

AAA. American Academy of Audiology. (2010). Clinical Practice Guidelines: Diagnosis, Treatment and Management of Children and Adults with Central Auditory Processing Disorder.https://audiologyweb.s3.amazonaws.com/migrated/CAPD\%20Guidelines\%208-2010. pdf_539952af956c79.73897613.pdf.

ASHA. American Speech-Language-Hearing Association. (2005) (Central) Auditory Processing Disorders - the role of the audiologist [Position Statement]. https: www.asha.org/policy.

BSA. British Society of Audiology. (2011). Practice guidance: An overview of current management of auditory processing disorder (APD). http://www.thebsa.org.uk/wpcontent/uploads/2014/04/BSA_APD_PositionPaper_31March11_FINAL.pdf.

Carlile, S., Martin, R., McAnally, K. (2005). Spectral information in sound localization. Int Rev Neurobiol., 70, 399-434.

CRFa. Conselho Federal de Fonoaudiologia. (2020). Guia de Orientação No 01/2020. "Dispõe sobre avaliação e intervenção no Processamento Auditivo Central". https://www.fonoaudiologia.org.br/comunicacao/guia-de-orientacao-avaliacao-eintervencao-no-processamento-auditivocentral/.

Dias, T. L. L. \& Pereira, L. D. (2008). Habilidade de localização e lateralização sonora em deficientes visuais. Rev Soc Bras Fonoaudiol. 13(4), 352-6.

Fonseca, C. B. F. \& Iorio, M. C. M. (2006). Aplicação do teste de lateralização sonora em idosos. Pró-Fono R. Atual. Cient. Barueri, 18(2), $197-206$.

Furst, M., Bresloff, I., Levine, R. A., Merlob, P.L. \& Attias, J. J. (2004) Interaural time coincidence detectors are present at birth: evidence from binaural interaction. Hear Res. 87(1-2), 63-72.

Geffner, D. (2019). Central auditory processing disorders: definition, description, Auditory processing and behaviors. In: Geffner, D. \& Ross-Swain, D. (Eds.). Auditory processing disorders: assessment, management, and treatment. Plural Publishing.

Gerente, J. G. S., Pascoal, A. G. \& Pereira, M. L. M. (2008). Localização espacial em indivíduos cegos congênitos: estudo comparativo da posição tridimensional da cabeça em adultos cegos congênitos e indivíduos videntes. Rev. Bras. 14(1), 111-120.

Gicov, R. A. (2015). Limiar Diferencial de Mascaramento em Crianças de Sete a Oito Anos. Equilíbrio Corporal Saúde, 7(1), 17-20.

Gil, A. C. (2002). Como Elaborar Projetos de Pesquisa. Editora Atlas.

Grote, B, Pecka, M. (2014). The natural history of sound localization in mam-mals - a story of neuronal inhibition. Front Neural Circuits. 8, 1-19.

Lewald, J. (2002). Opposing effects of head position on sound localization in blind \& sighted human subjects. Eur J Neurosci. 5, $1219-1224$.

Lima-Gregório, A. M. et al. (2010). Otite média recorrente e habilidade de localização sonora em pré-escolares. Rev. CEFAC, 12(6), 1033-40.

Luiz, C. B. L., Garcia, M.V., Perissinoto, J., Goulart, A. L. \& Azevedo M. F. (2016). Relação entre as habilidades auditivas no primeiro ano de vida e o diagnóstico de linguagem em prematuros. Rev. CEFAC. 18(6), 1316-1322.

Menezes, P. L., Andrade, K. C. L., Carnaúba, A. T. L., Cabral, F. B., Leal, M. C., Pereira, L. D. (2014). Sound localization and occupational noise. CLINICS. 69(2), 83-86.

Menezes, P. L. \& Motta, M. A. (2005). Audição animal. In: Menezes, P.L., Caldas Neto, S. \& Motta, M. A. (org). Biofísica da audição. Lovise, $141-149$. 
Research, Society and Development, v. 10, n. 8, e27910817333, 2021

(CC BY 4.0) | ISSN 2525-3409 | DOI: http://dx.doi.org/10.33448/rsd-v10i8.17333

Menezes, P. L., Oliveira, A. C., Moraes, L., Rocha, L. P. \& Lima, V. P. (2004). Ressonância: um estudo da orelha externa. Pró-Fono. 16(3), 333-40.

Menezes, P. L., Soares, I. A., Caldas Neto, S., Maciel, R. \& Motta, M. A. (2003). Estudo da localização sonora em ouvintes normais. J Bras Fonoaudiol. 4(15), 109-13.

Mondelli, M. F. C. G., Jacob, R. T. S., Ribeiro, J. P., Felici, M. G. F. M. \& Sanches, R. C. P. (2010). Unilateral hearing loss: The benefit of auditory localization after adaptation of hearing aids individual. Art. Int. Otorrinolaringol. 14(3), 309-15.

Musiek, F. E., Baran, J. A. (2020). The Auditory System: anatomy,physiology and clinical correlates. San Diego, CA: Plural Publishing.

Nishihata, R., Vieira, M. R., Pereira, L. D. \& Chiari, B. M. (2012). Processamento temporal, localização e fechamento auditivo em portadores de perda auditiva unilateral. Rev Soc Bras Fonoaudiol. 17(3), 266-73.

Nix, J., Hohmann, V. (2006). Sound source localization in real sound fields based on empirical statistics of interaural parameters. J Acoust Soc Am. 19(1), 46379.

Oliveira, A. C., Rocha, L. P., Lima, V. P., Morais Netto, L., Menezes P. L. \& Colafêmina, J. F. (2008a). Localização de fontes sonoras a importância das diferenças dos limiares auditivos interaurais. Rev. Soc. Bras. Fonoaudiol. 3(1), 7-11.

Oliveira, A. C., Morais Netto, L. S., Rocha, L. P., Lima, V. P. \& Menezes, P. L. (2008b). Variações anatômicas na localização sonora. Rev CEFAC. São Paulo. 10(3), 385-391.

Oliveira, A. P., Momensohn-Santos, T.M., Carmo, M. P. \& Fiore, A. (2020). Testes de fala no ruído uma revisão integrativa. Distúrb Comum. São Paulo. 32(1), 124-139.

Pereira, L. D. \& Schochat, E. (2011). Testes auditivos comportamentais para avaliação do processamento auditivo central. Barueri (SP): Pró-Fono, p.16.

Pereira, A. S., Shitsuka, D. M., Pereira, F. J., Shitsuka, R. (2018). Metodologia da Pesquisa Científica. Universidade Federal de Santa Maria, ed. 1, Santa Maria.

Ramos, C. S., Pereira, L. D. (2005). Processamento auditivo e audiometria de altas freqüências em escolares de São Paulo. Pró-Fono Revista de Atualização Científica, Barueri (SP). 17(2), 153-164.

Risouda, M., Hansona, J. N., Gauvrita, F., Renarda, C., Lemesrea, P. E., Bonnea, N. X. \& Vicenta, C. (2018). Sound source localization. European Annals of Otorhinolaryngology, Head and Neck diseases. 135(4), 259-264.

Souza, M. A., Passaglio, N. J. S., Souza, V. C., Scopel, R. R. \& Lemos, S. M. A. (2015). Ordenação temporal simples e localização sonora: associação com fatores ambientais e desenvolvimento de linguagem. Audiol Commun Res. 20(1), 24-31.

Tharpe, A. M. (2007). Unilateral hearing loss in children: A mountain or a molehill? The Hearing Journal. 60(7), 10-16. 